Revue de droit comparé du travail et de la sécurité sociale

3 | 2019

Les migrations internationales de travail

\title{
Les travailleurs migrants temporaires en Australie
} Joanna Howe

\section{OpenEdition}

Journals

Édition électronique

URL : https://journals.openedition.org/rdctss/1480

DOI : $10.4000 /$ rdctss. 1480

ISSN : 2262-9815

\section{Éditeur}

Centre de droit comparé du travail et de la sécurité sociale

\section{Édition imprimée}

Date de publication : 1 novembre 2019

Pagination : 124-135

ISSN : 2117-4350

\section{Référence électronique}

Joanna Howe, «Les travailleurs migrants temporaires en Australie », Revue de droit comparé du travail et de la sécurité sociale [En ligne], 3 | 2019, mis en ligne le 01 novembre 2021, consulté le 13 novembre 2021. URL : http://journals.openedition.org/rdctss/1480 ; DOI : https://doi.org/10.4000/rdctss.1480

\section{(c) (i) (9)}

Revue de droit comparé du travail et de la sécurité sociale est mise à disposition selon les termes de la Licence Creative Commons Attribution - Pas d'Utilisation Commerciale - Pas de Modification 4.0 International. 


\title{
LES TRAVAILLEURS MIGRANTS TEMPORAIRES EN AUSTRALIE
}

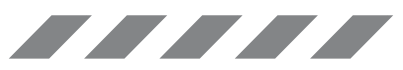

\begin{abstract}
This article considers the regulation of entry pathways for temporary migrants into the Australian labour market. Australia's temporary labour migration program accounts for one tenth of the labour market and has increased substantially in the last three decades. Although there is a discrete dedicated labour migration program for temporary skilled workers, there is also a substantial contribution made by unskilled and low skilled temporary migrants through the international student and working holiday maker programs.
\end{abstract}

KEY WORDS : Temporary Labour Migration, Labour Law, Exploitation, Work.

\section{RÉSUMÉ}

Cet article étudie la réglementation des voies d'entrée pour les migrants temporaires sur le marché du travail australien. Le programme australien de migration de main-d'œuvre temporaire représente un dixième du marché du travail et a considérablement augmenté au cours des trois dernières décennies. Bien qu'il existe un programme spécifique de migration de main-d'œuvre dédié aux travailleurs temporaires qualifiés, les migrants temporaires non qualifiés et peu qualifiés apportent également une contribution substantielle aux programmes d'étudiants internationaux et de vacanciers qui travaillent.

MOTS CLÉS: Migration de travail temporaire, droit du travail, exploitation, travail. 
approche juridique retenue par l'Australie pour réglementer les migrations de main-d'œuvre a radicalement changé depuis les années 90 . Si le programme australien d'immigration adopté après la Seconde guerre mondiale était fondé sur l'idéal d'une migration permanente, contribuant à l'édification de la nation et de la citoyenneté ${ }^{1}$, c'est finalement la migration temporaire des travailleurs qui est devenue la norme. Le nombre de travailleurs migrants temporaires a ainsi quintuplé depuis $1996^{2}$. Théoriquement, les visas attribués en cas de pénurie temporaire de maind'œuvre qualifiée et pour les nouveaux programmes du marché du travail destinés aux travailleurs du Pacifique sont les seuls à avoir un objectif spécifiquement professionnel. Or, dans la pratique, les étudiants étrangers et les touristes ont également le droit de travailler d'une part, et d'autre part une grande partie du travail considéré comme «non qualifié » ou " peu qualifié » est effectué par des migrants temporaires munis de ce type de visas ${ }^{3}$. La structure du programme temporaire influence le programme permanent. La migration temporaire est devenue une source essentielle de migrants permanents puisque la plupart des migrants qualifiés, titulaires de visas permanents, sont à l'origine entrés en Australie pour y travailler avec un statut de migrants temporaires ${ }^{4}$.

Il est généralement admis aujourd'hui que le programme australien de migration professionnelle temporaire crée un double marché du travail avec, d'une part la main-d'œuvre locale ayant le statut de citoyen et, d'autre part les travailleurs migrants temporaires. En comparant les niveaux de réglementation des visas de travail et des visas non professionnels, Howe et Reilly relèvent l'afflux de travailleurs migrants peu qualifiés qui se crée indirectement et le fait que la réglementation n'est pas en mesure de protéger

1 M. E. Crock, "Contract or Compact: Skilled Migration and the Dictates of Politics and Ideology", Georgetown Immigration Law Journal, vol.16, 2001, p. 133.

2 En 1996-1997, 25786 visas de sous-catégorie 457 ont été octroyés, contre 125070 en 2011 2012. Voir J. Phillips, H. Spinks, «Skilled Migration: Temporary and Permanent Flows to Australia », Parliamentary Library, 2012, p. 40; "Trends in Migration: Australia 2010-2011», Australian Government, Department of Immigration and Citizenship, 2012, p. 37.

3 Migration Regulations 1994 (Cth), Australian Government, p. 79; A. Boucher, « Australia's De Facto Low Skilled Migration Programs », in Migration: The Economic Debate (Rapport de recherche du CEDA - Comité pour le Développement Economique de l'Australie), Novembre 2016, Chapitre 2.

4 Environ 50000 titulaires de visas 457 sont devenus des résidents permanents en 2015-2016, soit près de $40 \%$ du nombre total annuel de migrants qualifiés (permanents) australiens. Voir P. Mares, "The 457 Visa is Dead! Long Live the TSS? », Inside Story, 2017 : http://insidestory.org.au/the-457visa-is-dead-long-live-the-tss/ 
ces travailleurs 5 . Tham, Campbell et Boese désignent respectivement les visas professionnels et non professionnels comme des programmes de migration professionnelle « dédiés » et "de fait ${ }^{6}$. Selon Forsyth, cette distinction génère un marché du travail à deux niveaux dans lequel des programmes dédiés et réglementés concernant le marché du travail représentent «le plus haut niveau », tandis que les filières de fait présentent " peu ou pas de cadre réglementaire concernant les besoins du marché du travail ou la protection contre l'exploitation » $^{7}$. Wright et Clibborn utilisent le terme de " portes » pour identifier le type et les conditions correspondant à chaque voie d'entrée des travailleurs migrants ${ }^{8}$. S'appuyant également sur l'idée des "portes», Howe, Charlesworth et Brennan étudient, dans une analyse distincte, la manière dont le contrôle et la sélection de l'immigration en Australie et en Nouvelle-Zélande ont été étayés par un développement sexué des compétences?. Chacune de ces études identifie la manière dont les règles en matière d'immigration contribuent à la segmentation du marché du travail en Australie.

Le présent article s'intéresse aux différentes voies d'entrée dans le cadre du programme australien d'immigration temporaire. Si la voie d'entrée officielle des travailleurs migrants en Australie est plus limitée, sujette à une réglementation plus stricte, les visas destinés aux étudiants et aux touristes étrangers constituent en revanche une voie non réglementée qui permet aux migrants temporaires d'exercer des professions très variées sur le marché du travail australien. II existe dès lors une nette différence entre les niveaux de réglementation, l'envergure et l'impact de ces différentes voies d'entrée.

La principale voie d'immigration des travailleurs est le visa accordé en cas de pénurie temporaire de main-d'œuvre qualifiée (Temporary Skill Shortage visa, TSS ci-après), qui se compose de deux filières. La première concerne les professions jugées très utiles pour l'économie australienne et sont alignées sur les stratégies à long terme du gouvernement en matière de formation et de main-d'œuvre. Cette filière permet d'obtenir un visa de 4 ans et offre une voie d'accès à la résidence permanente. La seconde, appelée «filière à court terme ", concerne les métiers pour lesquels il existe un besoin immédiat. Elle a été conçue comme une solution purement temporaire, avec un visa qui expire au bout de 2 ans et ne donne aucun accès à la résidence permanente.

5 J. Howe, A. Reilly, " Meeting Australia's Labour Needs: The Case for a New Low-Skill Work Visa », Federal Law Review, vol. 43, 2015, p. 259.

6 J-C Tham, I. Campbell, M. Boese, "Why is Labour Protection for Temporary Migrant Workers so Fraught?: A Perspective from Australia », in J. Howe and R. Owens (dir.), Temporary Labour Migration in the Global Era: The Regulatory Challenges, Hart Publishing, 2016, p. 173.

7 A. Forsyth, Victorian Inquiry into the Labour Hire Industry and Insecure Work, Industrial Relations Victoria, 2016, p. 316.

8 C. F. Wright, S. Clibborn, « Back Door, Side Door or Front Door? An Emerging De-Facto Low-Skilled Immigration Policy in Australia ", Comparative Labor Law \& Policy Journal, 2017, p. 165.

9 J. Howe, S. Charlesworth, D. Brennan, « Migration Pathways for Frontline Care Workers in Australia and New Zealand: Front Doors, Side Doors, Back Doors and Trap Doors », University of New South Wales Law Journal, 2019, vol. 42, nº 1. 
Comme son prédécesseur (le visa 457), le visa TSS ne permet pas la migration temporaire dans des métiers peu qualifiés. Il est conçu comme un visa de travail temporaire hautement qualifié. Cependant, le visa 457 a connu certaines périodes durant lesquelles les travailleurs migrants temporaires peu ou semi-qualifiés furent autorisés à entrer sur le marché du travail australien. En effet, le visa 457 a été "déréglementé ${ }^{10}$ au début des années 2000 et les employeurs ont alors pu recourir à des travailleurs peu qualifiés, malgré l'orientation officielle du programme favorisant les travailleurs migrants temporaires qualifiés ${ }^{11}$. Un dispositif d'accords régionaux s'en est suivi ${ }^{12}$, permettant aux employeurs de conclure des accords après avoir demandé à l'organisme régional de certification d'attester que «le poste ne pouvait être raisonnablement pourvu sur place $»^{13}$. Dès la fin 2002, une disposition prévoyait de supprimer les seuils de salaire et de compétences du visa 457 pour les accords régionaux, si bien que les employeurs étaient autorisés à ne verser à leurs travailleurs que $90 \%$ du salaire minimum requis ${ }^{14}$. Ils ont également été en mesure d'engager de la main-d'œuvre dans des métiers considérés comme peu qualifiés, à l'instar des " assistants de soins personnels», profession correspondant au niveau 6 de la classification australienne des professions ( $\mathrm{ASCO} »)^{15}$.

Dans un souci d'intégrité et en raison de son utilisation croissante pour des emplois peu qualifiés, des modifications importantes ont été apportées au visa 457 en 2008-2009, en vue de le réorienter vers un travail très qualifié. Cette initiative faisait suite à un certain nombre d'examens approfondis du programme ${ }^{16}$, chacun visant à éviter que le visa 457 ne s'éloigne de sa vocation initiale de programme d'immigration de travailleurs qualifiés. En 2008, un groupe d'experts externe sur les visas de la sous-classe 457 a indiqué que le

10 I. Campbell, J-C Tham, « Labour Market Deregulation and Temporary Migrant Labour Schemes: An Analysis of the 457 Visa Program », Australian Journal of Labour Law, 2013, vol. 26, p. 239.

11 J. Howe, "Is the Net Cast Too Wide? », Federal Law Review, 2013.

12 Migration Regulations 1994 (Cth), Australian Government, op. cit.

13 Australian Government, Department of Immigration and Citizenship, « Frequently Asked Questions: Permanent Employer Sponsored Program Reforms July 2012 »: https://rdanorthernrivers.org.au/ download/ industry and economic development/rsms/rsms-pes-faq.pdf. Le terme "régional» est défini au sens large. Les employeurs de Sydney, Newcastle, Wollongong, Melbourne, Brisbane, la Gold Coast et Perth ne peuvent conclure d'accord régional; B. Kinnaird, "Current Issues in the Skilled Temporary Subclass 457 Visa », People and Place, 2006, vol. 14, p. 49.

14 J. Phillips, «Temporary (Long Stay) Business Visas: Subclass 457 », Parliamentary Library, Note de recherche $n^{\circ} 15,2007$.

15 Australian Bureau of Statistics, "Australian Standard Classification of Occupations (ASCO) Second Edition » (n 1220.01997) : http://www.abs.gov.au/ausstats/abs@.nsf/0/7F887D870E0673BECA25697E00185133? Opendocument

16 Australian Government, Department of Immigration and Citizenship, «Final Report to the Minister for Immigration and Citizenship », 2008 : https://www.voced.edu.au/content/ngv:41113; «Temporary Visas ... Permanent Benefits: Ensuring the Effectiveness, Fairness and Integrity of the Temporary Business Visa Program », Comité permanent mixte sur les migrations, Parliament of Australia, 2007 ; B. Deegan, "Visa Subclass 457 Integrity Review », Rapport Deegan, 2008 : https:// archive.home affairs.gov.au/Workingin Australia/Documents/457-integrity-review.pdf 
visa 457 était devenu, par défaut, un «visa général pour recourir à de la main-d'œuvre », préconisant de ce fait son rééquilibrage en faveur de la migration temporaire de travailleurs qualifiés ${ }^{17}$. Ceci faisait écho à l'opinion exprimée en 2007 par le Comité permanent mixte sur les migrations, qui s'opposait fermement à l'utilisation du visa 457 pour faire face aux pénuries de main-d'œuvre non qualifiée, dans la mesure où cela «compromettrait son acceptabilité par la communauté en général $\aleph^{18}$. Par ailleurs, le Rapport Deegan préconisait également une compilation rigoureuse de listes de métiers faisant face à une pénurie de personnel pour vérifier la réalité d'une carence de main-d'œuvre qualifiée dans les métiers indiqués par les employeurs. Le but était d'éviter «le risque que ce type de visa ne soit détourné pour importer de la main-d'œuvre non qualifiée, ou pour importer de la maind'œuvre qualifiée malgré une offre locale abondante $»^{19}$.

Ces études ont incité le gouvernement fédéral à supprimer les accords régionaux conclus autour de visas 457 et la dérogation accordée aux employeurs sur le salaire minimum ${ }^{20}$, fermant dès lors une voie d'immigration légale pour les travailleurs de soins de première ligne. Les professions de niveau 5 à 7 de l'Australian and New Zealand Standard Classification of Occupations (ANZSCO ci-après) n'étaient donc plus éligibles à une migration professionnelle temporaire vers les régions. Les employeurs souhaitant avoir accès à des travailleurs migrants temporaires peu qualifiés devaient désormais y recourir par la voie des accords de travail. En outre, l'application d'une réduction du salaire minimum a été remplacée par l'exigence de suivre les salaires du marché et par des salaires planchers pour les travailleurs migrants temporaires titulaires d'un visa $457^{21}$. Une autre série de réformes a suivi en 2013, lorsque des tests menés par les employeurs sur le marché du travail ont été introduits pour certaines professions et que l'obligation de verser les salaires du marché a été renforcée ${ }^{22}$.

Ainsi, dans sa dernière version, remplacée ensuite par le TSS, le visa 457 autorisait uniquement l'entrée des professions de niveau 1 à 3 de l'ANZSCO et ne constituait donc pas une voie d'immigration temporaire pour les emplois peu ou moyennement qualifiés ${ }^{23}$. Cette approche a été poursuivie lors de l'introduction du visa TSS. Néanmoins, le visa 457 et son successeur, le visa TSS, permettent l'entrée de personnes titulaires de visas secondaires (partenaires et personnes à la charge du titulaire du visa principal) qui ne sont pas soumises

17 Visa Subclass 457 External Reference Group: Groupe de référence externe sur les visas de sousclasse 457 mis en place en 2008 (Minister for Immigration and Citizenship).

18 «Temporary Visas ... Permanent Benefits: Ensuring the Effectiveness, Fairness and Integrity of the Temporary Business Visa Program », Comité permanent mixte sur les migrations, op. cit., p. 12.

19 Rapport Deegan, op. cit., p. 34.

20 «Temporary Visas ... Permanent Benefits: Ensuring the Effectiveness, Fairness and Integrity of the Temporary Business Visa Program », op. cit., 2007, p. 10.

21 Pour une analyse de ces réformes, voir J. Howe, «The Migration Legislation Amendment (Worker Protection) Act 2008: Long Overdue Reform, but Have Migrant Workers Been Sold Short?", Australian Journal of Labour Law, vol. 23, 2010, p. 251.

22 Pour une analyse de ces réformes, voir J. Howe, "Is the Net Cast Too Wide? », op. cit.

23 A partir du 1er juillet 2010, le DIBP a commencé à utiliser les normes de l'ANZSCO à la place de celles de l'ASCO pour la classification des emplois. 
aux mêmes restrictions que le titulaire du visa principal et peuvent occuper n'importe quel type d'emploi. Bien que l'on ne dispose pas de données sur l'activité professionnelle des titulaires de visas secondaires, il est probable que certains d'entre eux occupent des emplois moyennement ou peu qualifiés.

Outre le TSS, le développement de nouveaux programmes d'immigration professionnelle pour les travailleurs du Pacifique fournit de nouvelles voies d'entrée qui, bien que restreintes, facilitent leur migration temporaire vers le marché du travail australien pour y occuper des emplois peu et semi-qualifiés. La raison d'être de ces nouvelles initiatives est d'élargir les possibilités d'emploi - en particulier pour les femmes - tout en développant les compétences, en augmentant les flux de transferts de fonds ${ }^{24}$, et en répondant aux besoins du marché du travail et de l'économie australienne. En 2016, un nouveau programme pilote de 5 ans a ainsi été mis en place. II offre jusqu'à 250 places aux travailleurs des micro-Etats du Pacifique, avec un visa de trois ans, le Microstate Pilot Visa (MPV ci-après) qui permet d'occuper des emplois peu qualifiés dans le nord de l'Australie ${ }^{25}$. Bien que ce projet pilote cible les emplois non saisonniers dans des secteurs tels que I'hébergement, le tourisme, l'hôtellerie et les soins aux personnes âgées, secteurs dans lesquels la pénurie de main-d'œuvre est reconnue, les titulaires de ces visas sont autorisés à travailler dans n'importe quel autre domaine ${ }^{26}$. Le premier groupe de travailleurs est arrivé en octobre 2016 et occupait des postes d'entretien ménager et d'intendance ${ }^{27}$. L'arrivée d'autres groupes de travailleurs provenant de ces micro-Etats grâce à de tels visas est d'ores et déjà programmée, et deux entreprises de soins aux personnes âgées, enregistrées auprès du Ministère des Affaires étrangères et du Commerce (DFAT), pourront employer ces travailleurs dans le cadre du programme (un troisième fournisseur de soins étant actuellement en pourparlers avec le DFAT ${ }^{28}$.

En septembre 2017, cette approche du développement régional et des défis du marché du travail en Australie a été élargie avec l'annonce d'un nouveau régime concernant la main-d'œuvre du Pacifique, le Pacific Labour Scheme (PLS ci-après), qui étend et formalise de différentes manières le régime du MPV. Le secteur des soins de santé est considéré comme le plus important des trois secteurs croissants couverts par le PLS ${ }^{29}$. Le PLS est un programme de parrainage par l'employeur qui a débuté en juillet 2018 avec l'objectif d'accueillir 2000 travailleurs de Kiribati, Nauru et Tuvalu, conformément à l'objectif du

24 Australian Government, «Our North, Our Future: White Paper on Developing Northern Australia » (Livre Blanc, juin 2015): http://northernaustralia.gov.au/files/files/NAWP-FullReport.pdf. Voir également Comité permanent mixte sur les migrations, "Seasonal Change: Inquiry into the Seasonal Worker Programme » (Rapport, Commonwealth d'Australie, mai 2016, 5 [2.8]).

25 Ibid., 6 [2.10], 13 [2.34].

26 RadioAustralia,PacificBeats, 2016(ConcettaFierravanti-Wells): http://ministers.dfat.gov.au/fierravantiwells/transcripts/Pages/2016/cf tr 161017.aspx?w=p2wUlmE1t7kKl1\%2BiOm3gqg\%3D\%3D

27 lbid.

28 Ibid.

29 Australian Government, Ministère des Affaires étrangères et du Commerce 2017 c. « Pacific Labour Scheme", Canberra. 
MPV qui vise à élargir les avantages de la migration de main-d'œuvre pour favoriser le développement des plus petits pays insulaires du Pacifique (les PIC). Le recrutement s'effectue via une nouvelle Pacific Labour Facility (PLF), qui permet au DFAT de jouer un rôle de médiateur entre les employeurs et les travailleurs des pays insulaires océaniens dans le cadre du programme d'accueil de travailleurs saisonniers et du recrutement du PLS. Etant donné que les phases de conception et de développement du PLS sont toujours en cours, il est difficile à ce stade d'évaluer l'importance de cette filière pour les travailleurs de première ligne du secteur des soins.

Outre la multitude de filières d'immigration temporaire, le programme permanent de l'Australie revêt également une grande importance, eu égard au nombre de travailleurs concernés et à la complexité de sa conception. Le programme d'immigration de l'Australie compte 190000 places, dont 128550 réservées aux émigrants qualifiés ${ }^{30}$. Les réfugiés migrants constituent une autre source potentielle de main-d'œuvre dans le programme permanent, bien que leur capacité à trouver et à garder un travail soit souvent limitée par le manque de contacts locaux, d'expérience professionnelle sur place et, dans certains cas, une maîtrise insuffisante de l'anglais ${ }^{31}$. Une fois qu'un demandeur d'asile a obtenu le statut de réfugié, il dispose d'un droit illimité à l'emploi en Australie.

En parallèle avec la filière standard des emplois qualifiés via le TSS puis des visas 457, il existe la voie des "accords de travail » qui permet aux employeurs d'embaucher des travailleurs n'ayant pas les compétences professionnelles ou linguistiques requises par la voie standard. La filière des accords de travail existe depuis le lancement du visa 457 en 1996. Son principal attrait pour les employeurs est la possibilité de recruter des travailleurs pour des emplois classés aux niveaux 4 à 7 de l'ANZSCO, ainsi que la possibilité de négocier des concessions sur les exigences réglementaires du visa 457 standard. Les accords de travail doivent assurer des conditions «non moins favorables " visant à garantir que les salaires et les conditions de travail du détenteur d'un visa 457 sont équivalents à ceux d'un travailleur australien pour un poste équivalent ${ }^{32}$, ainsi que la réalisation d'une enquête sur le marché du travail. Etant donné que les accords doivent être négociés individuellement sur plusieurs mois avec le ministère de l'Intérieur, il est prévu d'intégrer des garanties dans le processus d'élaboration de ces accords afin d'éviter que l'on prive les travailleurs locaux de leurs emplois ou que l'on exploite la vulnérabilité des travailleurs migrants temporaires. Toutefois, cette approche réglementaire n'est en aucun cas infaillible, car les associations d'employeurs continuent de demander la déréglementation et la simplification du processus des accords de travail, ce qui signifie que l'intégrité de la filière des accords de travail dépend du pouvoir discrétionnaire des ministres en ce qui concerne le cadre de négociation de ces accords ${ }^{33}$.

30 Commission sur la productivité, « Migrant Intake into Australia: Productivity Commission Inquiry Report », Rapport d'enquête $n^{\circ} 77,13$ avril 2016, p. 75.

31 Voir H. Graeme, "A Significant Contribution: The Economic, Social and Civic Contributions of First and Second Generation Humanitarian Entrants", Australian Government, Department of Immigration and Citizenship, 2011.

32 Migration Regulations 1994 (Cth), Australian Government, op. cit.

33 lbid. 
La décision, prise en 2017, de publier les noms des entreprises ayant passé des accords de travail et de mettre cette liste à jour chaque trimestre a constitué une étape importante, améliorant la transparence et la responsabilité de la filière des accords de travail ${ }^{34}$ à l'égard du public. Si l'utilité de ces informations reste limitée - puisque la page Web ne donne aucune information sur les concessions accordées, sur le nombre de travailleurs migrants dont l'entrée est autorisée en vertu de chaque accord de travail ou de leur lieu de travail - elles donnent néanmoins une indication sur le type d'industrie et d'employeurs qui ont recours à cette filière ${ }^{35}$.

Des accords de travail dans certaines régions australiennes sont également possibles grâce à un accord d'immigration pour une zone désignée (DAMA) ${ }^{36}$. Cela permet aux employeurs d'avoir accès à une main-d'œuvre migrante temporaire grâce à un accordcadre négocié avec un représentant de la zone désignée, tel qu'un conseil ou un organisme gouvernemental. Les employeurs peuvent demander au représentant régional de signer un contrat de travail régi par l'accord-cadre. Les directives du DAMA autorisent des concessions sur les exigences du régime des visas TSS en matière de salaire, de qualification et de maîtrise de l'anglais ${ }^{37}$. Alors que le visa TSS autorise uniquement l'entrée de travailleurs parrainés dans des métiers classés aux niveaux de compétence 1 à 3 de l'ANZSCO, le programme DAMA permet aux travailleurs d'être parrainés pour occuper des professions de niveau $4^{38}$. Le premier DAMA a été finalisé pour le Territoire du Nord et couvre un certain nombre d'emplois semi-qualifiés ${ }^{39}$. Etant donné que ces postes sont classés comme "vulnérables », aucune dérogation n'est autorisée au regard du niveau d'anglais exigé. Toutefois, le titulaire d'un visa DAMA exerçant l'un de ces emplois peut recevoir un salaire inférieur au seuil de revenu pour les immigrants temporaires qualifiés (TSMIT) ${ }^{40}$. Les travailleurs entrés sous DAMA sont soumis à un régime de contrôle des compétences plus strict que les

34 Voir J-C. Tham, "Law-Making and Temporary Migrant Labour Schemes: Accountability and the 457 Visa Scheme », Australian Journal of Administrative Law, 2009, vol. 17, p. 18. Pour un examen des problèmes de reponsabilisation et de transparence dans la sous-classe de visa 457, voir J. Howe, "Accountability and Transparency under the Subclass 457 Visa Program: Is There Cause for Concern? », Australian Journal of Administrative Law, 2014, vol. 21, p. 139.

35 Australian Government, Department of Home Affairs, "List of Current Labour Agreements", 12/11/2018: https://immi.homeaffairs.gov.au/visas/employing-and-sponsoring-someone/sponsoringworkerrs/ nominating-a-position/labour-agreements/list-of-current-labour-agreements

36 Australian Government, Department of Immigration and Border Protection, «Designated Area Migration Agreements ».

$37 \mathrm{lbid}$.

38 Ibid.

39 Migration Corporation of Australia, "Occupations Accessible under the DAMA», 2016: http:// www.migrationcorporation.com.au/wp-content/uploads/2016/04/04.2016-Occupationsaccessibleunderthedama. pdf

$40 \mathrm{lbid}$. 
travailleurs plus qualifiés. Une fois en Australie, ils doivent faire évaluer leurs compétences et prouver que leur expérience de travail est authentique. Leur employeur doit également remplir une déclaration solennelle confirmant que le titulaire du visa possède l'expérience professionnelle et les qualifications requises pour répondre aux exigences du poste ${ }^{41}$. Les employeurs sont tenus de s'adresser à l'administrateur DAMA pour parrainer des travailleurs et sont soumis à certaines exigences en matière d'examen du marché du travail même si ces dernières reposent en grande partie sur l'attestation par l'employeur d'une pénurie de main-d'œuvre. Aussi la rigueur de cette mesure est-elle quelque peu discutable ${ }^{42}$. Bien que les DAMA nécessitent l'approbation du gouvernement fédéral et que leur négociation, comme leur finalisation, requièrent beaucoup de temps, ce programme représente une filière d'immigration permettant aux travailleurs semi-qualifiés de travailler dans certaines régions d'Australie, sous réserve d'un certain nombre de conditions supplémentaires par rapport au régime standard des visas TSS.

Par ailleurs, il existe des visas non professionnels qui jouent un rôle important si l'on considère l'immigration dans le secteur des soins à la personne dans son ensemble, puisqu'un grand nombre des emplois dans ce secteur sont effectivement tenus par les titulaires de ce type de visas. Les visas vacances-travail (working holiday visas, sous-classes de visas 417 et 462) permettent ainsi aux migrants temporaires originaires de 41 pays, âgés de 18 à 30 ans, de travailler pendant leurs vacances et de rester durant un an en Australie $^{43}$. Les visas vacances-travail permettent de travailler pendant la totalité des 12 mois du visa, mais seulement 6 mois pour un même employeur ${ }^{44}$. En novembre 2005, la loi a été modifiée pour permettre aux titulaires de visas vacances-travail de demander un deuxième visa s'ils ont exercé pendant trois mois un "travail spécifique » dans les secteurs des mines, de la construction et de l'agriculture dans des zones rurales de l'Australie ${ }^{45}$. Bien que ce visa ait une vocation non professionnelle, le DIBP a reconnu les motivations professionnelles de ces étrangers en vacances. II rapporte que l'augmentation récente du nombre de vacanciers en activité "semble en grande partie être associée à la situation économique générale dans le monde » en 2011-2012, car les perspectives du marché du travail dans certains pays partenaires restent incertaines ${ }^{46}$. Le nombre de titulaires de ces visas a régulièrement augmenté depuis leur création ${ }^{47}$. L'intérêt accru des jeunes travailleurs

41 Migration Corporation of Australia, "DAMA FAQs - Factsheet 4: Market Rates »: http://www. migration corporation.com.au/wp-content/uploads/2015/01/DAMA-FAQs-1.pdf

42 J. Howe, "Does Australia Need an Expert Commission to Assist with Managing its Labour Migration Program? », Australian Journal of Labour Law, 2014, p. 233.

43 Australian Government, Department of Home Affairs, "Working Holiday Maker Visa Program Report», Rapport du 31/12/2017: https://www.homeaffairs.gov.au/research-and-stats/files/ working-holiday-report-dec17.pdf. L'Australie a également conclu des accords de visas vacancestravail avec l'Autriche, la République tchèque, I'Equateur, la Grèce et la Papouasie-Nouvelle-Guinée mais ces accords n'étaient pas entrés en vigueur au 31 décembre 2017.

44 Migration Regulations 1994 (Cth), Australian Government, op. cit. Voir également Department of Immigration and Citizenship, "Working Holiday Maker Visa Program Report», Rapport du $30 / 06 / 2013, n^{\circ} 4$.

45 Australian Government, Department of Immigration and Citizenship, « Working Holiday Maker Visa Program Report $», n^{\circ} 104$.

46 Ibid., $n^{\circ} 7$.

47 Australian Government, Department of Immigration and Border Protection, «Working Holiday Maker Visa Programme Report », 30/06/2015: https://www.homeaffairs.gov.au/research-and-stats/ files/working-holiday-report-june15.pdf 
taïwanais et sud-coréens pour l'obtention d'une prorogation d'un an du visa de vacancestravail pourrait être lié au fait que « le salaire minimum est considérablement plus bas dans les deux pays ${ }^{48}$, a souligné le Médiateur du travail équitable ${ }^{49}$. II est intéressant de noter que le Royaume-Uni, Taïwan et la Corée du Sud sont les trois principaux pays d'origine des vacanciers qui travaillent et qui demandent une reconduction de leur visa vacances-travail pour une année supplémentaire ${ }^{50}$.

Si les spécialistes ont souligné l'impact considérable sur le marché du travail des vacanciers qui occupent un emploi ${ }^{51}$, les rapports officiels du gouvernement ne cessent de rappeler que l'objectif de ce visa est un « échange culturel » et que « le travail est accessoire par rapport à l'objectif principal $\aleph^{52}$. Le Ministère de l'intérieur déclare dans ses directives que «le travail en Australie ne doit pas être l'objet principal de la visite du titulaire du visa $»^{53}$. II est donc significatif que, l'exposé des motifs pour l'ensemble de réformes de 2016 sur le visa «Working Holiday Maker» reconnaisse que ce visa a contribué sensiblement à pallier les pénuries de main-d'œuvre dans l'économie australienne ${ }^{54}$. On souligne ainsi la contribution de ces vacanciers au marché du travail, suggérant une évolution de la position des décideurs politiques. Par exemple, l'exposé des motifs indique que les réformes proposées «reconnaissent l'importance des vacanciers qui travaillent dans l'économie australienne ${ }^{55}$, le rôle du programme comme "source importante de main-d'œuvre supplémentaire $~^{56}$ et le rôle des titulaires de ces visas comme "moteurs de la croissance économique future $\aleph^{57}$. Cette reconnaissance officielle de la contribution professionnelle des vacanciers qui travaillent a été associée à des modifications leur permettant de travailler pour le même employeur pendant 12 mois dans deux régions distinctes.

48 Au 1er juillet 2015, le salaire minimum était de 5,06 AUD à Taïwan et de 6,69 AUD en Corée du Sud, contre 17,29 USD en Australie.

49 «Inquiry into the Wages and Conditions of People Working under the 417 Working Holiday Visa Program », Commonwealth d'Australie, 2016, p. 10.

50 Australian Government, Department of Home Affairs, "Working Holiday Maker Visa Program Report», op. cit., p. 7.

51 Migration Regulations 1994 (Cth), Australian Government, op. cit.; A. Reilly, "Low-Cost Labour or Cultural Exchange? Reforming the Working Holiday Visa Programme», Economic and Labour Relations Review, 2015, vol. 26, p. 474.

52 Australian Government, Department of Immigration and Citizenship, « Working Holiday Maker Visa Program Report », p. 3.

53 Australian Government, Department of Home Affairs, "What is the Working Holiday Maker Program? " : https://archive.homeaffairs.gov.au/lega/lega/form/immi-fags/what-is-the-workingholiday-maker-program

54 Income Tax Rates Amendment (Working Holiday Maker Reform) Act 2016 (Cth).

55 Mémorandum explicatif, Income Tax Rates Amendment (Working Holiday Maker Reform) Bill 2016 (Cth) $n^{\circ} 10[1.4]$.

56 Ibid., $n^{\circ} 43[3.12]$.

57 Ibid., $n^{\circ} 49$ [3.39]. 
Les étudiants étrangers sont autorisés à travailler à temps partiel pendant leurs études ${ }^{58}$. Depuis 2008, la possibilité de travailler est automatiquement incluse dans les visas étudiants alors qu'auparavant, il fallait en faire la demande. En 2013, les étudiants étrangers sont devenus éligibles à la sous-classe 485 des visas (temporaires) pour jeunes diplômés, ce qui leur offre de nouvelles opportunités de vie et de travail en Australie après la fin de leurs études. La libéralisation de l'autorisation de travailler pour les étudiants étrangers a pour objectif de donner à l'Australie un avantage concurrentiel sur les principales destinations choisies par les étudiants étrangers - les Etats-Unis, le Canada et le Royaume-Uni - où le droit de travailler n'existe pas ou n'est pas aussi étendu ${ }^{59}$.

Cet aperçu montre que le programme actuel de migration de main-d'œuvre en Australie comporte une multitude de voies d'entrée sur le marché du travail, chacune offrant aux titulaires de visas un statut, une sécurité et un statut variables. Auparavant, de nombreux migrants arrivaient avec le statut de migrants permanents. Aujourd'hui, une proportion croissante des travailleurs migrants nouvellement arrivés utilisent des filières de migration professionnelle temporaires et empruntent des voies latérales ou dérobées pour accéder au marché du travail australien. L'un des aspects les plus frappants de l'approche réglementaire australienne en matière de législation et de politique migratoires est le rôle prépondérant accordé aux employeurs dans la détermination de la structure des programmes d'immigration professionnelle temporaire et permanente, et la domination du modèle axé sur la demande après 1996. Deux canaux d'entrée (visas spéciaux pour les travailleurs du Pacifique et accords de travail) cherchent à répondre aux besoins en maind'œuvre de régions spécifiques ou d'employeurs particuliers en s'appuyant sur le modèle du parrainage par l'employeur.

Chacune de ces filières comprend des garanties réglementaires supplémentaires pour veiller à ce que la vulnérabilité des titulaires de visas qui empruntent ces voies soit prise en compte, bien qu'il ne soit pas certain que ces garanties protègent efficacement les travailleurs contre l'exploitation au travail et contre le non-respect du droit du travail australien. Deux autres canaux d'immigration professionnelle temporaire concernent des visas temporaires à vocation non professionnelle, mais n'accordent aucune protection supplémentaire sur le marché du travail pour tenir compte de la vulnérabilité de ces travailleurs. Les étudiants étrangers et les vacanciers qui travaillent occupent un nombre considérable d'emplois peu qualifiés et peu spécialisés en Australie. Leur place sur le marché du travail doit être prise en compte, en particulier à la lumière des récentes réformes concernant ces deux visas qui favorisent davantage la possibilité de travailler. De plus, les migrants récemment installés

58 Voir A. Reilly, "Protecting Vulnerable Workers: The Case of International Students", Australian Journal of Labour Law, 2012, vol. 25, p. 181.

59 Pour le Canada, voir Immigration and Citizenship, Gouvernement du Canada, « Work Off Campus as an International Student », 2017 : http://www.cic.gc.ca/english/study/work-offcampus.asp.

Pour le Royaume-Uni, voir UK Visas and Immigration, "Immigration Rules» (31/01/2012): https://assets.publishing.service.gov.uk/government/uploads/system/uploads/attachmentdata/ file/271060/29nov131.pdf.

Pour les Etats-Unis, voir A. Labi, « Britain's New Student Visa Policy Tightens Restricts Work but Not as Much as Feared », The Chronicle of Higher Education, 2011 : https://www.chronicle.com/article/ Britains-New-Student-Visa/126862

Pour l'Australie, voir Department of Home Affairs, Check Visa Details and Conditions: https://www. homeaffairs.gov.au/Trav/Stud/More/Work-conditions-for-Student-visa-holders 


\section{LES TRAVAILLEURS MIGRANTS TEMPORAIRES EN AUSTRALIE}

et les détenteurs de visas secondaires sont également présents sur le marché du travail australien.

Si l'on totalise le nombre de titulaires de visas dans chacune des filières migratoires australiennes susmentionnées, on comptabilise aujourd'hui plus d'un million de travailleurs migrants temporaires en Australie, bénéficiant des droits du travail et actifs sur le même marché que les travailleurs australiens locaux. Les immigrés temporaires représentent ainsi $1 / 10^{e}$ de la main-d'œuvre en Australie. Il est frappant de constater qu'il existe un certain nombre de voies d'entrée pour les migrants temporaires et que celles-ci sont soumises à différents niveaux de réglementation et de contrôle. Bien que l'immigration professionnelle temporaire officielle en Australie soit orientée vers l'accueil d'une main-d'œuvre migrante temporaire hautement qualifiée, il existe néanmoins une offre importante de travailleurs migrants peu qualifiés et semi-qualifiés, notamment grâce aux visas délivrés aux étudiants et aux vacanciers étrangers. Cumulativement, ces régimes de visas ont conduit à une forte dépendance des employeurs australiens vis-à-vis des travailleurs migrants temporaires.

\section{JOANNA HOWE}

Professeur agrégée de droit, Université d'Adélaïde.

Thématiques de recherche: Flux migratoires transnationaux, migration de travail temporaire, protection des travailleurs.

\section{Publications:}

J. Howe, (with S. Charlesworth \& D. Brennan) « Migration Pathways for Frontline Care Workers in Australia and New Zealand: Front Doors, Side Doors, Back Doors and Trap Doors », University of New South Wales Law Journal, 2019.

$\sim$ J. Howe, (with S. Le Mire) « Medical referral for abortion and freedom of conscience in Australian law », Journal of Law and Religion, 2019. 